\title{
Review \\ A three-dimensional model to analyze drug-drug interactions
}

\author{
Mark N. Prichard ${ }^{1}$ and Charles Shipman, Jr. ${ }^{1.2}$ \\ ${ }^{\prime}$ Department of Microbiology and Immunology, School of Medicine and ${ }^{2}$ Department of Biologic and \\ Materials Sciences, School of Dentistry, The University of Michigan, Ann Arbor, Michigan, U.S.A.
}

(Received 2 May 1990; accepted 26 July 1990)

\section{Summary}

Nearly four generations of investigators have studied combined drug effects. Their methods of generating and analyzing data have changed dramatically over the years but the basic problem has not. This review examines the inherent difficulties in analyzing combined drug effects and evaluates modern methods of describing these interactions. Researchers have traditionally used two-dimensional (2-D) methods to approximate the actual three-dimensional (3-D) nature of drug interactions. We conclude that these $2-\mathrm{D}$ methods are often inadequate when used to analyze synergistic and antagonistic drug interactions in antiviral and anticancer chemotherapy. We propose a direct and pragmatic 3-D approach to the problem, made possible by microcomputers and sophisticated graphics programs. This procedure directly elucidates the shape of the dose-response surface, identifies the regions of statistically significant synergy and antagonism, and quantitates these effects. It also greatly simplifies the problem since a 3-D surface presents complete drug interactions in a way that can be easily interpreted. We will show that understanding the shape of the resulting 3-D surface is essential to an understanding of complex drug interactions. This new method facilitates the rigorous analysis of drug-drug interactions and offers investigators powerful new tools to analyze combinations of antiviral and anticancer drugs.

Drug interaction; Three-dimensional; Chemotherapy; Synergy

Correspondence to: Charles Shipman, Jr., The University of Michigan, School of Dentistry, Department of Biologic and Materials Sciences, Ann Arbor, MI 48109-1078, U.S.A. 


\section{Introduction}

Although there is a need for continued development of new antiviral agents, the use of combinations of presently existing antiviral drugs is becoming increasingly important for a number of reasons (Freestone, 1985). For example, the use of drug combinations reduces the possibility of selecting drug resistant mutants. In addition, drug combinations which exploit synergistic interactions also may offer increased antiviral efficacy while decreasing cytotoxicity by minimizing the required therapeutic doses (Goldwin and Mantel, 1957; Jawetz, 1968; Kirsi et al., 1984; Sandstrom and Kaplan, 1987; Skipper, 1974; Steel and Peckham, 1979; or see review, Hall and Duncan, 1988). Not all combinations of drugs, however, synergistically inhibit viral replication. Some drugs when used in combination may even antagonize the individual antiviral effects (Voght et al., 1987). Because of these and other reasons, the accurate and complete evaluation of complex drug interactions is essential. When we examined the 2-D methods currently employed to analyze these interactions, however, we found that they were often inadequate. The 2-D methods are obliged to keep one variable constant and consequently are prone to miss or underestimate the important drug-drug interactions. Over 100 years ago, a very good theoretical 3-D model was developed and was characterized as the most accurate and complete way to describe these interactions (Loewe, 1953). Technological barriers in producing 3-D graphs, however, limited the practical application of this model. It is now possible, using computer assisted 3-D graphing techniques to bring this procedure into practice. This review details the inadequacies of the commonly used 2-D methods and presents a method using computer assisted $3-\mathrm{D}$ graphing techniques to bring the $3-\mathrm{D}$ techniques into practice.

\section{Development of analytical models}

\section{Dose-response surfaces and isobolograms}

A theoretical model for describing drug interactions was first proposed in 1872 by Fraser in reporting data describing the pharmacological antagonism between atropine and physostigmine. Since the experiments involved 3 variables, the respective drug concentrations and the biological effect, the most logical and concise way to plot the data was in three dimensions. Plotting the experimental data in this manner allowed the antagonism to be visualized by inspecting the shape of the 3-D surface. This work was significant in that drug-drug interactions were identified as a problem of three dimensions.

The 3-D model proposed by Fraser was not used extensively until more than 50 years later. Working with combinations of CNS stimulants and CNS depressants led Loewe, a German pharmacologist, to explore alternative ways of representing these complex interactions (Loewe, 1928, 1938, 1953, 1954, 1957). When experimental data were plotted with the drug concentrations on the $X$ and $Y$ axes and the resulting biological effect on the vertical $Z$ axis a surface was defined. Since this $3-D$ dose- 
response surface was analogous to the 2-D dose response curve used for drugs individually, its shape was considered to be an important characteristic of the drug interactions it described (Loewe, 1928). Theoretical dose-response surfaces for additive, antagonistic and synergistic interactions then were calculated, plotted and their characteristic shapes used to diagnose the type of interaction seen in subsequent experimental data (Loewe, 1953).

Technological limitations, however, made it extremely difficult to directly plot experimental data in three dimensions. Consequently, the idea of the isobol, or line of equal elevation, was borrowed from cartographers. This simple 2-D plot, called an 'isobologram', could be used to describe portions of the 3-D surface. The significance of these 2-D representations is that part of the 3-D dose-response surface and thus the type of interaction could be predicted from its shape. Since isobolograms are 2-D, one variable must be held constant if they are to describe a 3-D event. Typically, isobolograms are plotted at a fixed effect level of 50\%, much as drug activity for individual drugs is described by the $50 \%$ inhibitory concentration $\left(\mathrm{IC}_{50}\right)$.

Loewe identified the shapes of dose-response surfaces and isobolograms for additive, synergistic and antagonistic drug interactions. Additive drug interactions are defined by a set of horizontal lines intersecting the dose-response curves for the individual drugs. This surface yields linear horizontal cross sections or linear isobolograms (Fig. 1A). Synergistic drug interactions have a greater than additive biological effect, and thus have a convex shape like a billowing sail. A horizontal cross section of this surface yields a curved isobologram with the concave side up (Fig. 1B). Antagonistic drug interactions produce a lesser than

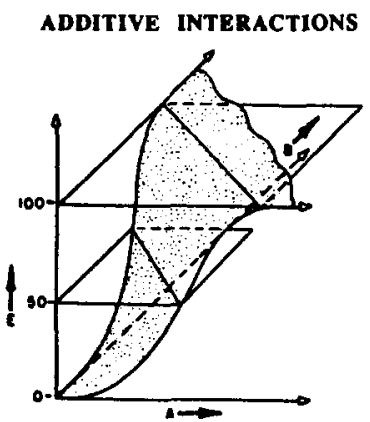

A

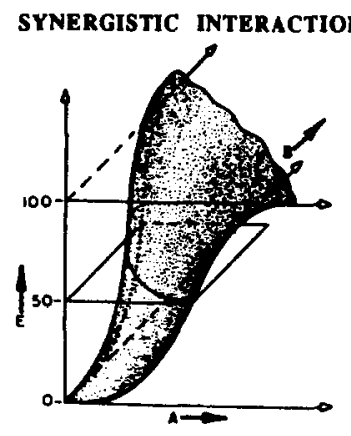

B
ANTAGONISTIC INTERACTIONS

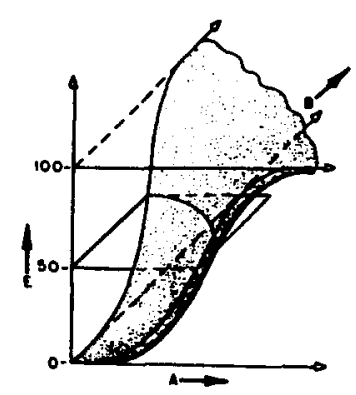

C

Fig. 1. Theoretical 3-D dose-response surfaces for drug combinations that interact (A) additively, (B) synergistically, and $(C)$ antagonistically. The $X$ and $Y$ axes represent the concentrations of the drugs $A$ and $B$ respectively with the vertical $Z$ axis representing the measured biological effect $E$. Dose-response surfaces for synergistic and antagonistic drug interactions billow up and sag down, respectively as compared to the additive surface. The intersections of the horizontal planes at the $50 \%$ effect level and the dose-response surfaces are equivalent to conventional isobolograms. The isobologram for purely additive interactions is linear as shown in panel $\mathrm{A}$. The isobolograms for drug combinations that are synergistic or antagonistic are shifted to the left or to the right of the linear additive isobologram respectively as depicted in panels B and C. [Reproduced from Loewe, S. (1953)]. 
expected response and consequently yield a surface which is concave like a sagging sail. Isobolograms derived from this surface are curved with the convex side up (Fig. 1C). The characteristic shapes of the isobolograms were subsequently used to visualize synergistic and antagonistic drug interactions instead of plotting the complete dose-response surface.

The calculations for plotting isobolograms required a strict definition of additivity. This is necessary because synergistic interactions were defined as being greater than the predicted additive interactions and antagonistic interactions were defined as being less than the predicted additive interactions. The theoretical additive effects were defined by Loewe as simply the sum of the individual effects:

$1=\frac{D_{1}}{\left(\mathrm{IC}_{\mathrm{p}}\right)_{1}}+\frac{D_{2}}{\left(\mathrm{IC}_{\mathrm{p}}\right)_{2}}$

with $D_{1}$ and $D_{2}$ equal to the doses of drugs 1 and 2 required in combination to reach an effect of $P$ percent and $\left(\mathrm{IC}_{\mathrm{p}}\right)_{1}$ and $\left(\mathrm{IC}_{\mathrm{p}}\right)_{2}$ equal to the doses required for each drug to individually produce an effect of $P$ percent. The solutions to this equation are all the combinations of concentrations of drugs 1 and 2 that yield an effect of $P$ percent and are equal to unity in this equation. Any result in this equation which is $<1$ or $>1$ would be indicative of synergy or antagonism, respectively. The $2-\mathrm{D}$ plot of all the concentrations that yield a $50 \%$ effect is equivalent to a horizontal cross section through the dose-response surface at the $50 \%$ effect level. This is the classical isobologram.

Loewe subsequently found, however, that experimental results did not always behave as the theoretical models predicted. Subsequent analyses of drug interactions revealed that drugs could act antagonistically at low effect levels $\left(\mathrm{IC}_{30}\right)$ and synergistically at high effect levels (IC 90 ) (Loewe, 1957). In addition, the same isobologram may even indicate both antagonism and synergy in different regions of the curve (Gessner, 1988; Berenbaum, 1988). These results reveal the inherent problems of using a 2-D method to describe a 3-D event. It may be necessary to plot isobolograms at many effect levels to accurately describe the interactions of a two drugs.

Despite its shortcomings, the isobologram concept was intuitively attractive and in 1954, the isobologram was independently rediscovered by Gertrude Elion and coworkers (Elion et al., 1954) and has been used extensively for many years to evaluate drug interactions in many fields (Ahmed and Tyrell, 1986; Allen et al., 1982; Fraser-Smith et al., 1985; Huggins et al., 1984; Johnson and Attanasio, 1987; Spector et al., 1985; Streifel and Howell, 1981; Webb, 1963). Recently, increased interest in the isobologram concept has brought about significant improvements in isobologram methodology: Isobolograms have been estimated from mathematical models (Carter et al., 1988) and analyzed statistically (Berenbaum, 1988; Gessner, 1988). This method of analysis has remained a standard in the fields of antiviral and anticancer chemotherapy for more than 25 years. Its ability to show simultaneously the effect at many different drug concentrations makes it a very valuable tool. 
In 1954, Bauer introduced a new way to present experimental data of drug combinations. Dose response curves for the first drug were plotted with several constant concentrations of the second drug. The resulting multiple dose-response curves were easily interpretable and were equivalent to taking vertical cross sections through the dose-response surface parallel to the axis of the first drug. This method of plotting is simple and is used often to make initial qualitative determinations of drug interactions (Bauer, 1954; Jackson, 1984; Johnson and Attanasio, 1987; MacKay, 1981). An example of data displayed in this manner is seen in Fig. 2.

In the late 1950's, two excellent review articles examined methods for analyzing drug-drug interactions (Goldwin and Mantel, 1957; Veldstra, 1956). At that time, many important questions regarding the nature of specific drug interactions remained unanswered because the relationships involved were too complex to be elucidated by the methods of analysis then available. There also was disagreement about how synergy should be defined. A careful review of the literature revealed seven different criteria used by authors when they wished to prove synergistic relationships (Goldwin and Mantel, 1957).

\section{Fractional inhibitory concentrations}

In 1963, the problem of how additivity should be defined was approached

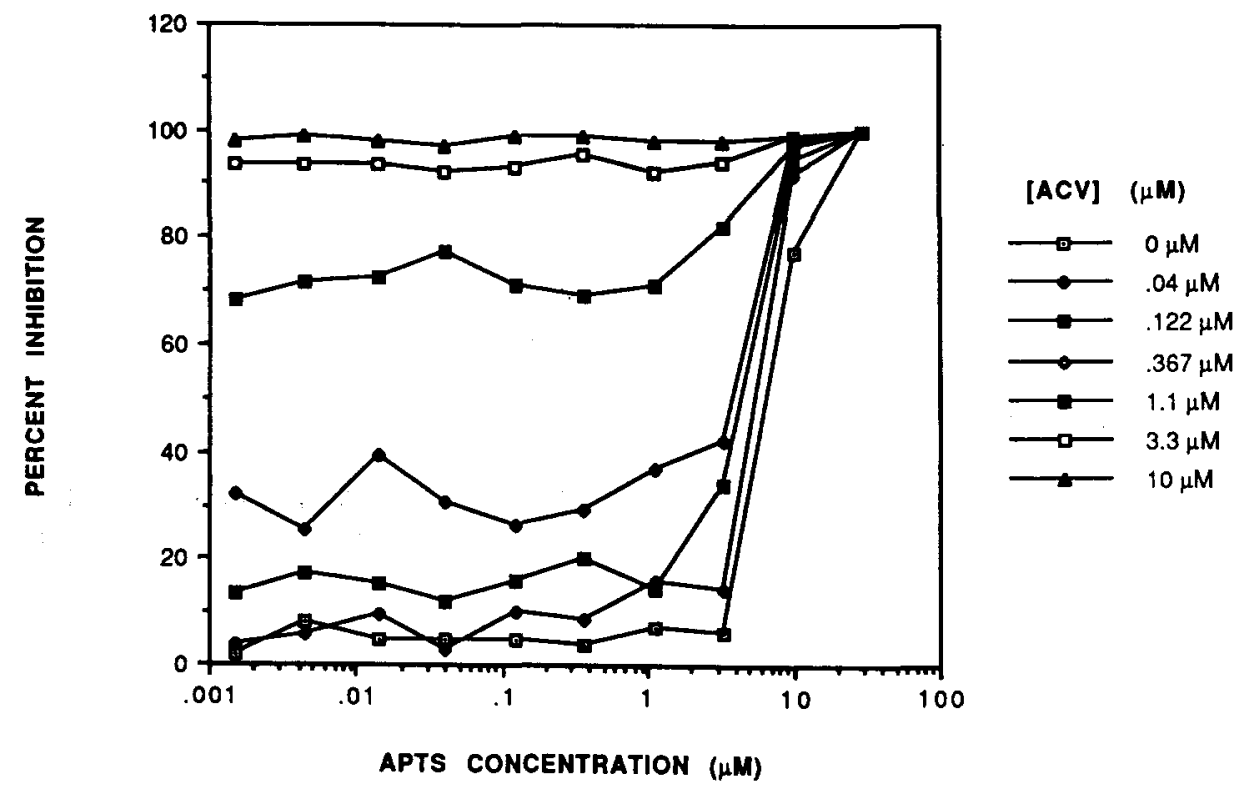

Fig. 2. Multiple dose-response curves representing the antiviral interactions between 2-acetylpyridine thiosemicarbazone (APTS) and acyclovir (ACV). APTS dose-response curves are plotted with the addition of concentrations of ACV ranging from $0.04 \mu \mathrm{M}$ to $10 \mu \mathrm{M}$. 
from the perspective of classical kinetics by Webb. Loewe's assumption, that the combined effect is the sum of the individual effects, was thought to be unrealistic because it did not reflect the reduction of the unaffected population by the first drug. The fractional products equation corrected for this discrepancy and was proposed as a more accurate way to calculate additivity:

$I_{1,2}=I_{1}+I_{2}-I_{1} I_{2}$

with $I_{1}$ and $I_{2}$ equal to the inhibition produced by drugs 1 and 2 alone, and $I_{1,2}$ equal to the inhibition of both drugs combined. This new equation was used to calculate additivity and like Loewe's equation would be equal to unity if the interactions were additive and $<1$ or $>1$ if the interactions were synergistic or antagonistic, respectively.

In a 1976 review article about drug interaction in immunosuppression, Berenbaum discussed the fractional product method proposed by Webb and the isobologram method proposed by Loewe. Berenbaum noted that whereas Webb's equation and Loewe's equation yielded solutions which could apparently quantitate synergy and antagonism they did so only at one arbitrary point. The data also were not easily interpretable when many different combinations of concentrations were used. The isobolograms, however, which were plotted from solutions to these equations, displayed simultaneously results for many drug concentrations which could be easily interpreted by inspecting the shape of the curve.

\section{Envelope of additivity}

The problem of precisely defining synergy in mathematical terms was specifically addressed by Steel and Peckham (1979). Additivity was calculated using both Loewe's and Webb's equations and the results were simultaneously plotted as isobolograms, thus forming an 'envelope of additivity' between the two isobolograms generated from the two equations. In this way, an investigator could conclude that synergy or antagonism was taking place if the experimental isobologram did not fall between the two additive isobolograms.

\section{Combination index}

Another mathematical approach to precisely define additivity was outlined in the 1980's by Chou and Talalay (Chou and Talalay, 1980, 1983, 1984). As Webb had done, Chou and Talalay approached the problem from the perspective of classical enzyme kinetics. A very general equation, which they called the 'median effect equation', was used for linearizing the sigmoid shaped dose-response curves and was defined as:

$f_{\mathrm{a}} / f_{\mathrm{u}}=\left(D / D_{\mathrm{m}}\right)^{\mathrm{m}}$

with $f_{\mathrm{a}}$ and $f_{\mathrm{u}}$ equal to the fractions affected and unaffected, respectively, by 
the dose $D$, with $D_{\mathrm{m}}$ as the dose required to elicit the median effect, and with $m$ as the coefficient of sigmoidicity. The median effect equation was used as a diagnostic tool to determine whether the inhibitors were mutually exclusive or mutually nonexclusive (Chou and Talalay, 1980; Palatini, 1982). This information identified the appropriate equation of additivity which was subsequently used to calculate the combination index $(\mathrm{CI})$ which is defined as:

$\mathbf{C I}=\frac{\left(f_{\mathrm{a}}\right)_{1,2}}{\left(f_{\mathrm{u}}\right)_{1,2}}=\frac{\left(f_{\mathrm{a}}\right)_{1}}{\left(f_{\mathrm{u}}\right)_{1}}+\frac{\left(f_{\mathrm{a}}\right)_{2}}{\left(f_{\mathrm{u}}\right)_{2}}$

for combinations of mutually exclusive inhibitors and,

$\mathrm{CI}=\frac{\left(f_{\mathrm{a}}\right)_{1,2}}{\left(f_{\mathrm{u}}\right)_{1,2}}=\frac{\left(f_{\mathrm{a}}\right)_{1}}{\left(f_{\mathrm{u}}\right)_{1}}+\frac{\left(f_{\mathrm{a}}\right)_{2}}{\left(f_{\mathrm{u}}\right)_{2}}+\frac{\left(f_{\mathrm{a}}\right)_{1}\left(f_{\mathrm{a}}\right)_{2}}{\left(f_{\mathrm{u}}\right)_{1}\left(f_{\mathrm{u}}\right)_{2}}$

for combinations of mutually nonexclusive inhibitors, with $\left(f_{\mathrm{a}}\right)$ and $\left(f_{\mathrm{u}}\right)$ equal to the fractions affected, and fractions unaffected, respectively, with subscripts designating the drug or combination of drugs used. These equations yield solutions of unity when additive interactions are present, and $<1$ or $>1$ when synergy or antagonism is present, respectively. The authors, however, did not use isobolograms to plot these results. The combination index was plotted on the $\mathrm{Y}$ axis, and the effect level, or the percent affected, was plotted on the $\mathrm{X}$ axis to display the results graphically (Fig. 3).

It is important to note that, in contrast to the authors' claims (Chou and Talalay, 1984), the CI equation for mutually exclusive inhibitors (eq. 4) is not equivalent to the equation proposed by Loewe (eq. 1, see appendix). To illustrate

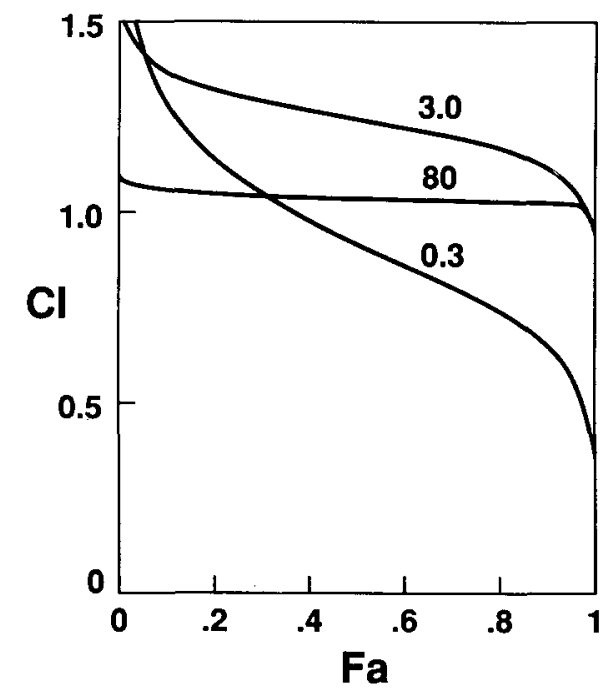

Fig. 3. Combination Index plot of the interactions between APTS and ACV plotted for ACV:APTS molar ratios of $0.3,3.0$ and 80 . Synergy is seen at a molar ratio of 0.3 , antagonism at a ratio of 3.0 , and additivity is seen at a ratio of 80 . 
A

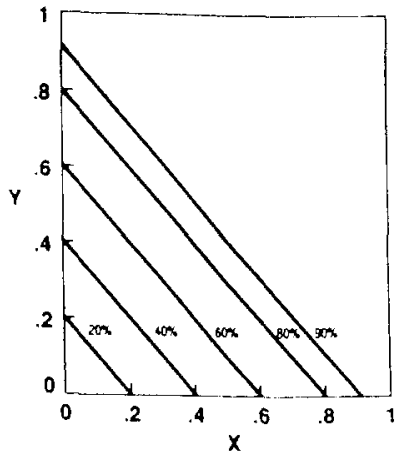

B

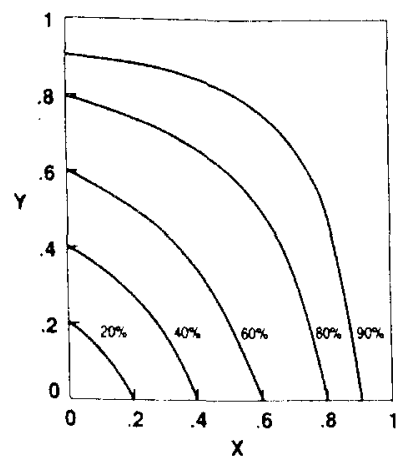

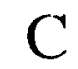

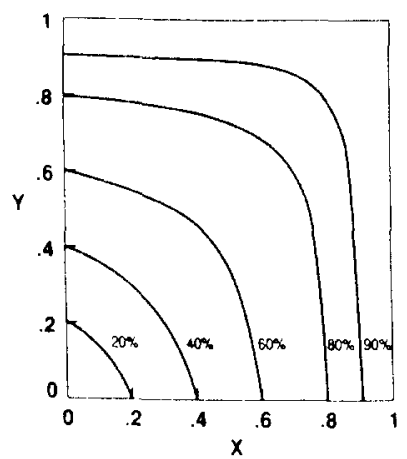

Fig. 4. Isobolograms of the theoretical additive dose-response surfaces calculated from two linear dose-response curves using the heteroadditive (similar site) assumption (A), the mutually nonexclusive (dissimilar site) assumption (B), and the mutually exclusive assumption (C). Any experimental points lying above or below this calculated surface would be indicative of synergy or antagonism respectively.

the differences between the preceeding equations, theoretical additive effects for two linear dose-response curves were calculated using Loewe's assumption (eq. 1), Webb's or the mutually nonexclusive assumption (eqs. 2 and 5) and the mutually exclusive assumption (eq. 4) and plotted as isobolograms (Fig. 4A-C). The predicted additivity using the three assumptions is not the same. For example, a combination that looks purely additive using the mutually nonexclusive assumption would look synergistic using the mutually exclusive assumption and antagonistic using Loewe's assumption. It is therefore important to understand and differentiate the three assumptions of additivity.

\section{The assumptions of additivity}

The equations for calculating theoretical additivity proposed by Webb, Loewe, and Chou and Talalay use different assumptions of additivity. These assumptions were used to derive equations which detected synergy and antagonism (eqs. 1,2,4 and 5). The equations used by the authors, however, contained only two variables. Any complete description of the phenomenon of multiple drug combinations must take the independent and dependent variables into consideration. In the case of a combination of two drugs three variables exist; the concentrations of the two drugs and the resulting biological effect. Since this event is defined by three variables, a 3-D equation is necessary to describe it completely. When a third variable was introduced into each equation discussed above, a simple equation could be derived which represented the basic assumption of additivity used by each of the authors. Three basic assumptions of additivity were identified. The differences between the three assumptions of additivity are illustrated by the following examples.

If a particular concentration of drug $X$ yields a $40 \%$ reduction of some biological effect, and a particular concentration of drug $Y$ also yields a $40 \%$ reduction of 
this effect, then one could expect that if these two drugs were used together, an $80 \%$ reduction would occur $(40 \%+40 \%=80 \%)$. This assumption, designated heteroadditivity by Loewe (eq. 1), appears naive at first, but experiments in which one drug was used in mock-combination with itself reveal precisely this kind of additivity (Palatini, 1982; Skipper, 1974). This type of additivity occurs when two inhibitors bind to a similar site on a common enzyme thus precluding the binding of a second inhibitor molecule. We have designated this assumption as the similar site assumption of additivity. The equation defining additivity of similar site (SS) inhibitors was derived from equation 1 by introducing a third variable (see appendix). The SS equation of additivity can be described as:

$$
Z=X+Y
$$

In this equation $Z$ is equal to the total inhibition produced by the combination of drugs $X$ and $Y$, with $X$ and $Y$ representing the inhibition produced by drugs $X$ and $Y$ alone respectively.

A more realistic case originally proposed by Webb asserts that inasmuch as drug $X$ reduces the unaffected population by $40 \%$, the addition of drug $Y$ should affect the remaining $60 \%$ by $40 \%$ for a total of $64 \%\{40 \%+[(40 \% \times(100 \%-40 \%)\}$. This case was confirmed experimentally when two drugs affected dissimilar sites on a target enzyme or enzymes (Chou and Talalay, 1983; Lam, 1987). Consequently, the assumption of additivity in this case we designate as the dissimilar site assumption of additivity. The equation for dissimilar site (DS) inhibitors is derived in the appendix from eq. 2 and 5 and can be described as:

$$
Z=X+Y(1-X)
$$

The equation for mutually exclusive inhibitors (eq. 4) proposed by Chou and Talalay (Chou and Talalay, $1980 ; 1983 ; 1984$ ) was reported to be equivalent to Loewe's assumption of additivity (eq. 1). This is not the case, however, (see appendix) and is of unknown biological significance.

The existence of two assumptions of additivity complicates analytical models as the two equations of additivity predict different additive effects and thus have different criteria for diagnosing synergy and antagonism. Using Loewe's isobologram method, only additivity using the SS assumption (eq. 6) would produce linear isobolograms as predicted by his model, and then only if the individual doseresponse curves were linear (Loewe, 1938). Isobolograms predicting additivity using the DS additive assumption (eq. 7), are not linear. The additive isobolograms for SS and DS inhibitors using two linear dose-response curves illustrate this point (Fig. 4A,B).

Since the SS and DS assumptions of additivity calculate additivity differently, their definitions of synergy, or greater than additive interactions, are necessarily different. It is therefore necessary to make assumptions about the mechanisms of the individual inhibitors. The most rigorous criteria for diagnosing synergy are defined by the SS assumption (eq. 6). The requirements for diagnosing synergy are 
significantly lower when the DS assumption is used (eq. 7). The order of stringency is naturally reversed for the diagnosis of antagonism with the DS assumption being the most stringent and the SS assumption being the least stringent.

It has been suggested that the DS assumption of additivity may not be valid for sigmoidal dose-response curves (Berenbaum, 1981, 1985). The author reasoned that if doses of two drugs produce the same inhibition individually, then half of the doses of both drugs used in combination should produce the same degree of inhibition. Using this criterion, a mock combination of a drug versus itself was shown to look synergistic when the DS assumption was used. This example, however, is not appropriate because the DS assumption of additivity should be used only when the drugs used in the combination affect different sites. This is particularly inappropriate in the above example because identical targets are being affected. Although isobolograms which predict additivity are typically not linear using the SS and DS assumptions they are nonetheless mathematically and theoretically valid. The SS and DS assumptions of additivity do not depend upon drug concentration and consequently are valid irrespective of the shape of the individual dose-response curve. Although there is some disagreement about how to define additivity, the addition of biological effects makes the fewest assumptions as it is a descriptive rather than a prescriptive method.

\section{Summary of current analytical methods}

The development of a precise mathematical definition of additivity, and thus synergy and antagonism, has proven essential in understanding drug interactions. It is equally important, however, to present experimental data in a concise manner which reflects the nature of the events portrayed. In the case of a two drug combination, a 3-D plot is most appropriate as it reflects the inherent 3-D nature of drug interactions. Very few investigators, however, present their data in this manner, presumably because of the technical difficulties in constructing 3-D graphs (see, however, Fraser, 1872). The 2-D and 1-D methods used by most researchers to approximate the nature of the interactions involved are inherently incomplete and give an oversimplified and often misleading picture of the experimental data. In the subsequent section of this review, methods currently used to evaluate drug interactions will be discussed and compared with the 3-D model.

\section{Isobolograms}

The isobologram method, while the oldest, is probably one of the best methods currently used as it is based on the concept of the 3-D dose-response surface. Using procedures outlined later, isobolograms also can be plotted directly from the 3-D dose-response surface. Isobolograms also can be constructed by plotting all combinations of concentrations of the two drugs which produce the chosen endpoint. The isobolograms are easy to interpret as they reveal how the drugs interact at many different combinations simultaneously. One limitation of this 
method is that isobolograms can only be used to look at combinations at one endpoint, such as combinations which give a $50 \%$ or a $90 \%$ reduction in virus replication. This problem, however, may be overcome by simultaneously plotting isobolograms at many different endpoints. The resulting plot was called a multiple isobologram by Loewe and an example can be seen in Fig. 5 (Loewe, 1957). Another disadvantage of this method is that isobolograms for additive interactions are only linear if the individual dose-response curves are linear. Typical sigmoid dose-response curves yield isobolograms which are concave down at low endpoints and concave up at higher endpoints. As a result, the diagnosis of synergy or antagonism from nonlinear isobolograms may not always be valid.

\section{Multiple dose-response curves}

The multiple dose-response curve method is a good method for preliminary evaluations of experimental data (Bauer, 1954; Johnson and Attanasio, 1987; MacKay, 1981). It is similar to the multiple isobologram approach, except the plot is equivalent to vertical cross sections through the dose-response surface parallel to the axis of the first drug. The results, however, are more difficult to interpret than those from the multiple isobologram as only one drug concentration is varied per line.

\section{Fractional products}

Webb's fractional product method, is as valid as the isobologram method de-

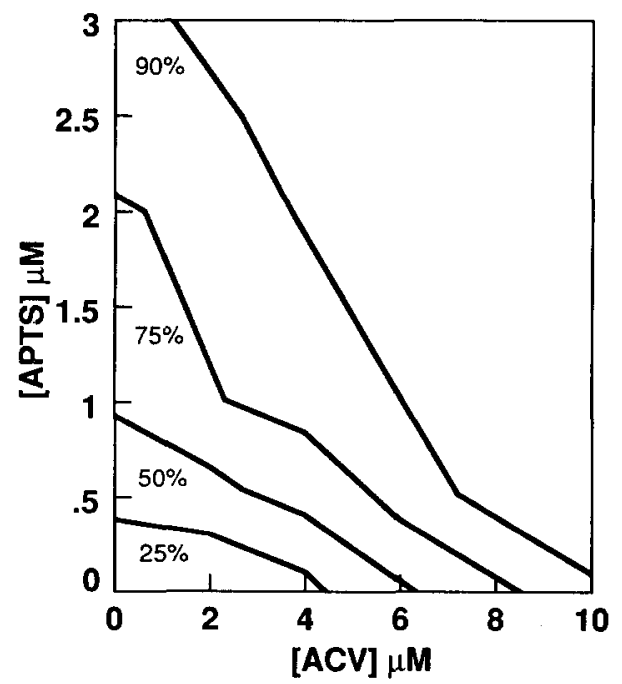

Fig. 5. Multiple isobologram of the antiviral interactions between APTS and ACV. The isobologram at the $25 \%$ endpoint indicates antagonism, the isobologram at the $50 \%$ endpoint indicates additivity, and isobolograms above the $50 \%$ endpoint indicate synergy. 
scribed above if it is plotted in isobologram form. Unfortunately this method is often misused with the researchers giving the numerical results from eq. 2 as the only evidence for synergistic interactions (Chan and Zabransky, 1987). The resulting number describes only one point on the dose response surface, and in many cases can indicate synergy, additivity, or marked antagonism depending on what combination the investigator arbitrarily chooses. A conscientious researcher should be skeptical of any results reported only in this manner.

\section{Envelope of additivity}

The envelope of additivity method is a variation of the traditional isobologram method and may reliably indicate synergy or antagonism even when the exact nature of the inhibition is unknown. This method is able to do this because it calculates and plots theoretical additivity in an isobologram format using both the SS and DS assumptions (Steel and Peckham, 1979). Any experimental data which do not fall within the envelope of additivity, created by the two additive isobolograms, can be diagnosed as synergy or antagonism depending on their positions. This method, like the isobologram method, only examines an arbitrarily selected endpoint and thus does not consider other levels of the dose-response surface.

\section{Combination index}

Chou and Talalay's combination index method, which has become increasingly popular in recent years (Potmesil et al., 1986; Schinazi et al., 1986), has two shortcomings. First, the equation used by the authors to describe mutually exclusive inhibitors (eq. 4) is not equivalent to the isobologram equation (eq. 1) (Fig. 4A-C, and see appendix). Therefore, results derived from eq. 4 are not equivalent to traditional isobolograms. Second, the experimental design requires fixed ratios of the two drugs. This experimental design is not as efficient as the previously proposed checkerboard dilution protocol (Mead and Pike, 1975). As fixed drug ratios examine effects along diagonal lines across the dose-response surface, data gathered in this way could reveal synergy, antagonism or additivism depending on the drug ratios chosen by the investigator (see Fig. 3). It is possible to describe a dose-response surface with several drug ratios, but the combined results in our opinion are difficult to interpret.

\section{Three-dimensional analysis}

Three-dimensional models and models of higher orders, can now be described using 3-D graphing techniques and mathematical equations (Solana et al., 1986). These methods, collectively called response surface methodology (RSM), are useful in many aspects of biological research and have been used to optimize growth media, and enzyme assay conditions (Carter et al., 1985; London et al., 1982; Maddox and Richert, 1977; Mead and Pike, 1975). RSM is useful whenever more 
than two variables are involved. We propose the application of these techniques to the study of drug interactions. This 3-D approach eliminates the problems inherent in the incomplete 2-D approximations proposed by other authors. A similar but less analytical 3-D method was published after this manuscript was prepared (Sühnel, 1990). In addition, another 3-D method called COMBO is being developed by Bunow and Weinstein (John N. Weinstein, personal communication).

The experimental methods which we have developed facilitate the routine analysis of drug combinations. Our experimental design is based on a checkerboard matrix of the permutations of serial dilutions of each of the drugs, including the drugs used individually. This experimental approach is not used by all investigators, but it is essential that it be employed for the accurate determination of all the interactions involved (Mead and Pike, 1975). Our method can also be used to analyze in vivo data if a similar experimental design is employed. The quantity of data required to perform this analysis is not necessarily large. A simple analysis can be performed on as few as 9 data points in a 3 by 3 matrix which is comparable to the data requirements for the other methods discussed. A larger data set, however, increases the resolution of this assay. We typically obtain excellent resolution with 80 data points in 8 by 10 matrices.

The generated data are subsequently transformed to calculate additive interactions with the DS or SS equations. This theoretical additive surface is subsequently subtracted from the experimental 3-D surface to reveal regions of synergy and antagonism. The calculations required for performing this analysis are quite simple. We recently have used a Macintosh ${ }^{\mathrm{TM}}$ computer (Apple Computer, Inc., Cupertino, CA) with the spreadsheet Microsoft ${ }^{\mathrm{TM}}$ Excel (Microsoft Corp, Redmond, WA), to perform the required calculations and the graphics program Deltasoft ${ }^{\mathrm{TM}}$ (Deltapoint Inc., Monterey, CA) to plot the data with excellent results. Any good statistics and graphics package with 3-D matrix plotting capabilities, however, should be capable of performing this simple analysis. This flexibility minimizes costs by utilizing software typically available in most laboratories. Herein we illustrate an analysis with the statistics and graphing program Statgraphics ${ }^{\mathrm{TM}}$ (STSC, Inc. Rockville, MD).

\section{Antiviral enzyme immunoassay}

The antiviral enzyme immunoassay (EIA) used is similar to those previously described (Landini and Baldassarri, 1983; Langlois et al., 1986; Rabalais et al., 1987 ) and is ideally suited to generate the large data sets required by this protocol. BS-C-1 (African green monkey kidney) cells, passaged in minimum essential medium with Earle's salts [MEM(E)] supplemented with $10 \%$ calf serum, 100 $\mathrm{U}$ penicillin $\mathrm{G}$ per $\mathrm{ml}$, and $100 \mu \mathrm{g}$ streptomycin sulfate per $\mathrm{ml}$, were seeded at a final concentration of 10000 cells per well in 96-well microtiter plates (Costar, Cambridge, MA). The cells were allowed to adhere for $24 \mathrm{~h}$ at $37^{\circ} \mathrm{C}$ in a humidified $4 \% \mathrm{CO}_{2}-96 \%$ air atmosphere. The medium then was removed and columns $2-12$ were infected with $100 \mu \mathrm{l}$ of the KOS strain of herpes simplex virus type 1 (HSV-1 KOS) at a multiplicity of infection of 0.01 . The virus used in these experiments 
was subcloned to ensure a homogeneous virus stock (Klein, 1975). Following a 1 $\mathrm{h}$ adsorption period, $100 \mu \mathrm{l}$ of a $4 \mathrm{X}$ solution of the first drug was added to the last columns of all plates including the uninfected cytotoxicity plate. Twofold serial dilutions of twice the desired final drug concentration were then performed across columns 3-11, leaving column 12 as a control for the second drug alone. $2 \mathrm{X}$ two fold serial solutions of the second drug were made in rows $A-G$ of a separate 96-well plate and subsequently transferred to the corresponding rows in the plates containing the dilutions of the first drug, with row $\mathrm{H}$ serving as a control for the first drug used alone. No drugs were added to columns 1 and 2 which were the cell and virus controls, respectively. After a $48 \mathrm{~h}$ incubation period, the medium was shaken out and $200 \mu \mathrm{l}$ of $95 \%$ ethanol were added to each well to fix the cell sheet. The ethanol was removed after $10 \mathrm{~min}$ and the plates were dried and frozen at $-76^{\circ} \mathrm{C}$ or stained immediately.

An EIA was then performed directly in the wells containing the infected cell sheets. The wells were first blocked with $200 \mu \mathrm{l}$ of $10 \%$ calf serum in Hepes buffered saline (HBS, Shipman, 1969) with 0.05\% Tween-20 (HBS-T) for $1 \mathrm{~h}$ at $37^{\circ} \mathrm{C}$. The plates were then shaken out and washed with HBS-T. $50 \mu \mathrm{l}$ of a 1:400 dilution of horseradish peroxidase conjugated rabbit anti-HSV-1 antibodies (Dako Corp. Santa Barbara, CA) in HBS supplemented with $10 \%$ calf serum, were then added to each well. Following a $1 \mathrm{~h}$ incubation, the plates were washed 4 times with HBS-T. The plates were subsequently developed with $100 \mu \mathrm{l}$ of a substrate solution consisting of $0.1 \mathrm{M}$ carbonate buffer, $\mathrm{pH} 5.0,0.005 \%(\mathrm{v} / \mathrm{v}) 30 \% \mathrm{H}_{2} \mathrm{O}_{2}$ and $1 \mathrm{mg} / \mathrm{ml} o$-phenylenediamine dihydrochloride (OPD, Sigma Chemical Co., St. Louis, MO). The reaction was stopped with $5 \mu$ l of $5 \mathrm{~N} \mathrm{H}_{2} \mathrm{SO}_{4}$ and read at 490 $\mathrm{nm}$ in a microplate kinetics reader (Biotek Instruments, Inc., Winooski, VT).

\section{Cytotoxicity determinations}

Simultaneous cytotoxicity controls were done as above except that the plates were not infected with virus. The cell growth was quantitated by staining the cell sheet with crystal violet, eluting with $1 \%(\mathrm{v} / \mathrm{v}) \mathrm{HCl}$ in ethanol, and reading at 570 $\mathrm{nm}$ in the microplate reader (Barer et al., 1986; Forti et al., 1986; Yeh et al., 1982).

\section{Data transformation}

The graphics and statistics computer program, Statgraphics ${ }^{\mathrm{TM}}$ (STSC, Inc. Rockville, MD), used in our protocol offers a number of powerful techniques to help researchers visualize the features of the generated 3-D surfaces. Experimental dose-response surfaces can be generated easily by the computer, and the vantage point may be changed to examine any region desired. Sections through the surface may also be displayed in the form of a contour plot, which is in actuality a multiple isobologram. Statgraphics ${ }^{\mathrm{TM}}$ also is capable of performing direct matrix transformations which are useful in manipulating the generated surfaces. Calculated additive effects may be subtracted from the experimental surfaces to identify regions of unexpected antiviral activity. 
The resulting antiviral and cytotoxicity data were either directly entered into Statgraphics ${ }^{\mathrm{TM}}$ or graphically transformed to a linear scale and then entered. The latter step was necessary only if the original data were in $\log _{10}$ form and conventional isobolograms were desired. Isobolograms were generated by directly plotting the linearized data matrix in the RESPONSE SURFACE MODE of Statgraphics ${ }^{\mathrm{TM}}$. The resulting dose response surface was either plotted in three dimensions, or in the form of a contour plot at any specified endpoints.

The most powerful method for analyzing these interactions, however, involved calculating theoretical additive effects directly from the individual dose-response curves determined in the assay. The calculated theoretical additivity was then compared to the experimental dose-response surface (Fig. 6A), and subsequently subtracted to reveal any areas of aberrant interaction. The calculations of theoretical additivity were performed with two matrices. The first matrix, $\mathrm{X}$, was a matrix consisting of the dose-response curve for drug $X$ repeated ten times. The resulting surface represented the predicted inhibition of the drug in all wells of the experimental plate and appears as replicates of the first dose-response curve plotted in three dimensions (Fig. 6B). The second matrix, Y, consists of the drug $\mathrm{Y}$ dose response curve eight times in a row. The resulting surface represents inhibition by drug $\mathrm{Y}$ in all wells of the experimental plate (Fig. 6C). Note that the $\mathrm{X}$ axes of the two dose-response surfaces are perpendicular to each other as in the experimental plot.

The two matrices described in the previous paragraph were then transformed using one or both of the equations for calculating additivity (eqs. 6,7). These calculations yielded matrices which represented the calculated antiviral effects if the combination was additive. These equations are easier to use than the equations proposed by other authors (eqs. 1-5) because they calculate additivity in its proper 3-D context. The resulting surface (Fig. 6D), which represents the calculated additivity of the drug combination, was then subtracted from the experimental surface (Fig. 6A) and plotted in 3-D and contour form to reveal any regions of synergy or antagonism (Figs. 6E,F, respectively). These plots represent deviations from expected interactions and would reveal a horizontal plane at $0 \%$ if the interactions were purely additive. Synergistic drug interactions would appear as a peak above the plane with a height corresponding to the percent above calculated additivity. Antagonistic interactions would appear as a pit with a negative value signifying the percent below the calculated additivity. The volumes of synergistic or antagonistic regions are equal to the relative quantity of synergy or antagonism produced per change in the two drug concentrations.

Data from the cytotoxicity plates were directly entered into a matrix in Statgraphics ${ }^{\mathrm{TM}}$ and plotted in the RESPONSE SURFACE MODE of this program. If there was no observable toxicity from the individual drugs, the plotted surface should appear as a horizontal plane that intersects the $\mathrm{Z}$ axis at $100 \%$ of cell growth. This surface represents cell growth and any deviation from this pattern is easily detectable. Synergistic cytotoxicity would appear as a pit in this plane and was seen when zidovudine and ganciclovir were used in combination (Prichard and Shipman, unpublished observations). Conversely, antagonistic cytotoxicity or one drug decreasing the cytotoxicity of another drug should appear as a peak on the surface. 

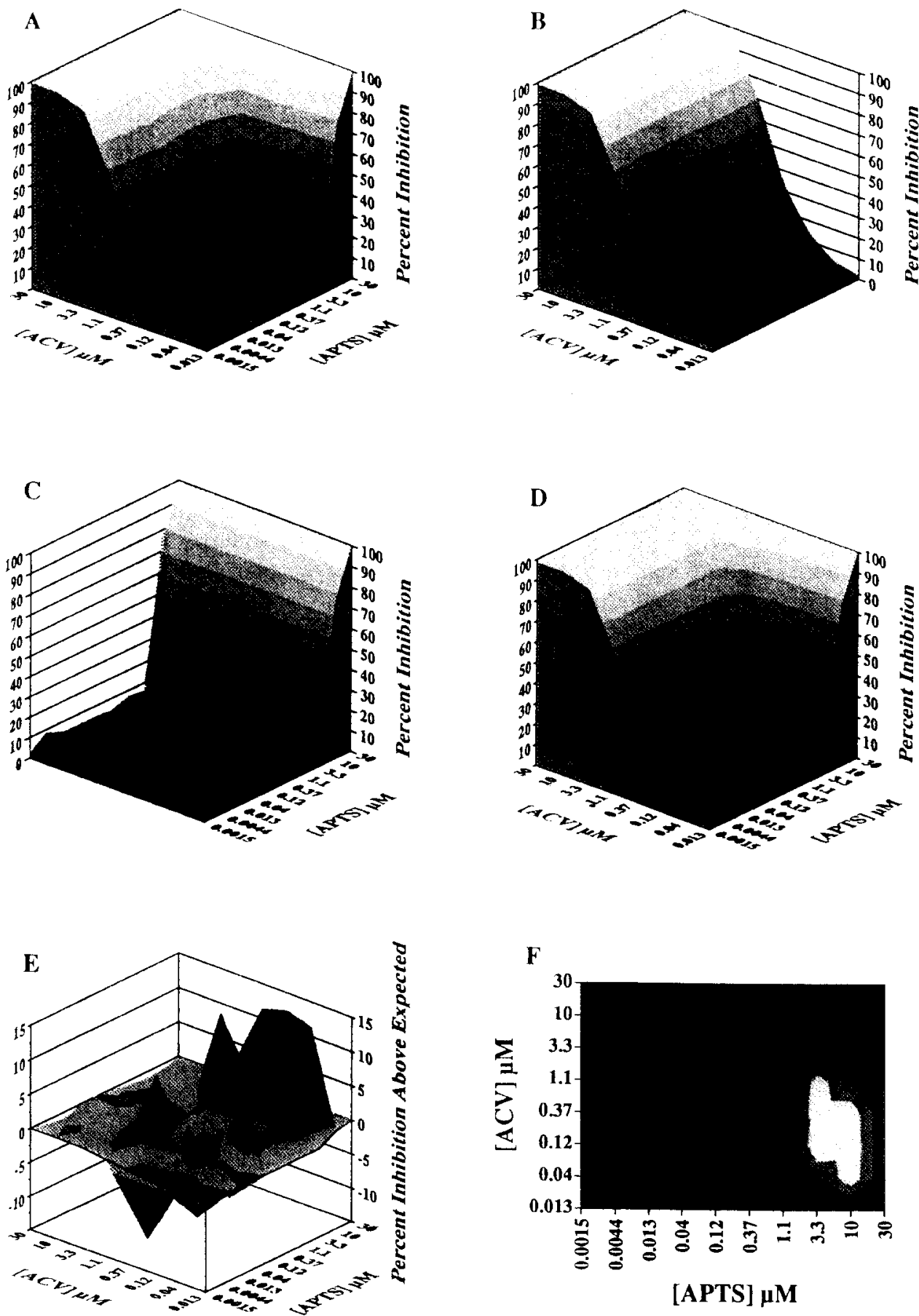

[APTS] $\mu \mathrm{M}$ 
The matrices resulting from transformations in Statgraphics ${ }^{\mathrm{TM}}$ can be printed. To enhance the graphics, the matrices were entered into Deltagraph ${ }^{\mathrm{TM}}$ to produce and print high quality figures.

\section{Statistical analysis}

Statgraphics ${ }^{\mathrm{TM}}$ also was used in the statistical analysis of the data. The data were subjected to analysis of variance (ANOVA), and the intraclass correlation coefficient was subsequently calculated (Hunt, 1986) to quantitate the error associated with the EIA antiviral assay. The error due to variance between replicates was summarized with a box and whisker plot (Fig. 7.). To determine the significance of differences between the experimental and calculated additive effects, the upper and lower 95\% confidence limits of the experimental data were compared to the calculated additive effects. If the lower confidence limit of a point was greater than the calculated additivity, the observed synergy was considered to be significant. Similarly, if the upper confidence limit was lower than the calculated additivity, the observed antagonism was considered to be significant.

\section{An example comparing methods of analysis}

The synergistic antiviral interactions between acyclovir (ACV), an inhibitor of HSV DNA polymerase, and inhibitors of the HSV encoded ribonucleoside diphosphate reductase (ribonucleotide reductase) are well documented (Karlsson and Harmenberg, 1988; Spector et al., 1985; Spector et al., 1989). The specific interactions, however, are quite complex and illustrate the shortcomings of existing 2-D methods for analyzing drug-drug interactions. In contrast, 3-D analysis revealed the complex interactions involved. The example examined here is the combination of acyclovir (gift of Burroughs Wellcome Co., Research Triangle Park, NC) and the ribonucleotide reductase inhibitor 2-acetylpyridine thiosemicarbazone (APTS, Turk et al., 1986a). The data were generated with the antiviral EIA and were analyzed by the methods described here.

Fig. 6. Three dimensional analysis of the antiviral interactions between APTS and ACV including the stages in the data transformation required to produce synergy plots. The experimental dose-response surface is represented in panel A. This surface is not convex as the drug concentrations are plotted on a log scale. Two matrices representing the antiviral effects of ACV (B), and APTS (C) alone were used to calculate the theoretical additivity using the dissimilar site (DS) assumption. The resulting calculated additive surface (D) was then subtracted from the experimental surface (A), yielding the synergy plot in surface (E) and contour plot (F) form. Statistically significant synergy and antagonism are represented by the white and black regions in panel $F$, respectively. 
The first method used to examine these interactions was the method of multiple dose-response curves (Fig. 2). Synergy at higher concentrations of ACV is revealed by the shift in the dose-response curves. This 2-D plot, however, was unable to statistically verify or quantitate the synergy present.

\section{Multiple isobologram}

The second method used to analyze these interactions was a multiple isobologram (Fig. 5). The isobologram plotted at the endpoint of $25 \%$ was concave down and suggested antagonistic interactions. The isobologram plotted at the $50 \%$ endpoint was essentially linear and suggested additive interactions. Isobolograms plotted at higher endpoints indicated synergistic antiviral interactions by being concave up. Thus, from this plot it appeared that the drug interactions involved were concentration dependent; the most potent synergy is present at higher endpoints and higher drug concentrations.

\section{Combination index $(C I)$}

The third type of analysis demonstrated here is the combination index method (Fig. 3). The analysis shown here was based on the mutually nonexclusive additive (DS) assumption because ACV and APTS are known to act on different targets. The results from the $\mathrm{CI}$ plot, like the results from the multiple isobologram, also suggested that the drug interactions were concentration dependent. The combination index plot demonstrated potent synergy at endpoints above $40 \%$ at a ACV:APTS molar ratio of 0.3 , but antagonism at a molar ratio of 3.0. Interestingly, at a molar ratio of 80 where the most intense antagonism was subsequently observed, the CI plot appeared additive. This apparent aberration is a direct result of the CI computer program dropping the first data point during the analysis. These results demonstrated that the CI also can be misleading and should be plotted at many different molar ratios to reveal the interactions involved. Even if multiple molar ratios are plotted, however, it is difficult to obtain any coherent relationship among them.

\section{Three-dimensional analysis}

The fourth method of analysis, the 3-D method (Fig. 6A-F), revealed that both synergistic and antagonistic antiviral interactions occur. This method of analysis was based on the dissimilar site assumption of additivity. The apex of synergy is seen at concentrations of APTS and ACV of approximately $4 \mu \mathrm{M}$ and $0.1 \mu \mathrm{M}$ respectively. Antagonistic interactions were also noted with a nadir at concentrations of APTS and ACV of $0.005 \mu \mathrm{M}$ and $0.4 \mu \mathrm{M}$, respectively. The 3-D method of analysis clearly demonstrates the concentration dependence of the drug-drug interactions and identifies the relationship between them.

ANOVA was performed on all data and yielded an intraclass correlation coefficient of $92.4 \%$ indicating that only $7.6 \%$ of the variation was within replicates. 


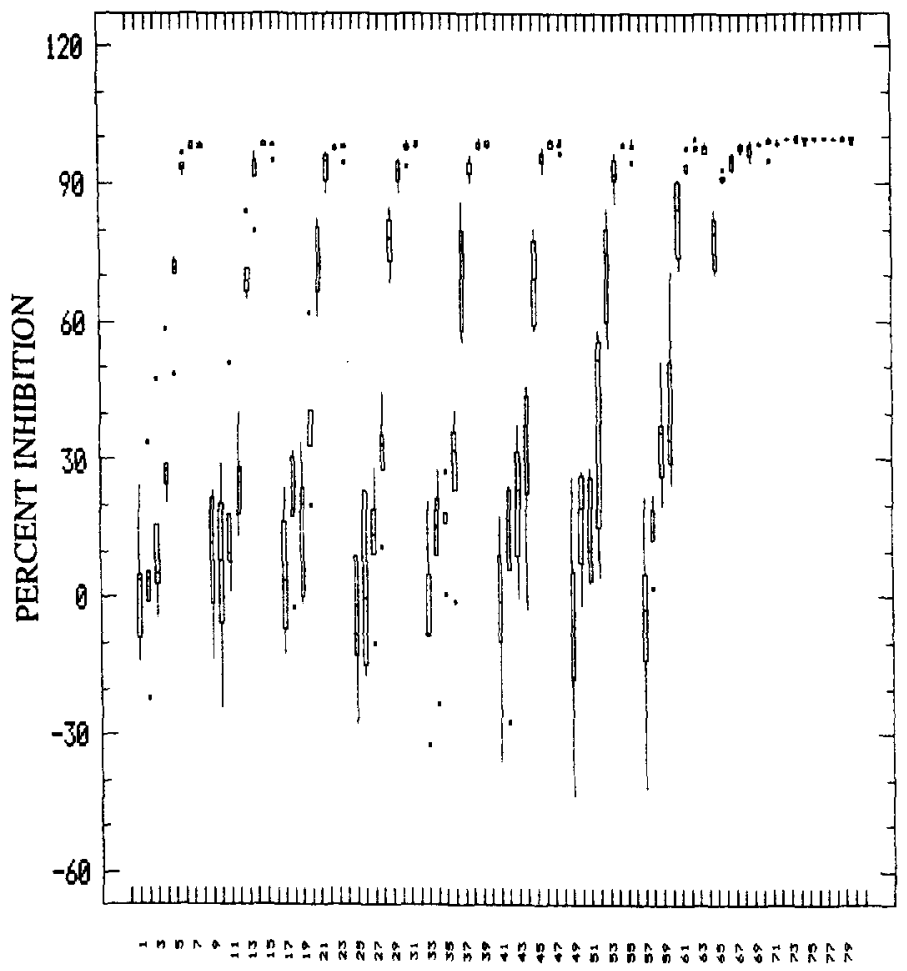

POINT NUMBER

Fig. 7. Multiple box and whisker plot of experimental data. The data point number corresponds to vertical cross sections parallel to the $Y$ axis starting at the left side. The box represents the middle 2 quartiles with the whiskers indicating the range of experimental values.

Error due to variance of replicates is shown in the box and whisker plot (Fig. 7). The data points correspond to vertical cross sections parallel to the $\mathrm{Y}$ axis with the box representing the middle two quartiles and with the mean marked within the box. The whiskers represent the range of values and outliers are expressed as individual points. The regions of synergy and antagonism identified were statistically significant as determined by the $95 \%$ confidence limits. Statistically significant synergy and antagonism are depicted as the white and black regions in Fig. 6F, respectively.

The rigorous 3-D method of analysis reveals why other methods of analysis were ineffective in clearly displaying the complex antiviral interactions between the two drugs. Both synergistic and antagonistic antiviral interactions are present in this particular combination and are dependent on the concentrations of both ACV and APTS. Because of this concentration dependence, 2-D methods are often unable to disclose the complex interactions, and either antagonism or synergy could be revealed depending upon the selection of variables.

When the data were analyzed with isobolograms, discreet endpoints had to be chosen and held constant for the isobolograms to be plotted. In this case, weak 
antagonism was seen at an endpoint of $25 \%$ and synergy was seen at endpoints of $75 \%$ and above. This is a result of isobolograms at low endpoints and high endpoints intersecting regions of antagonism and synergy, respectively, as demonstrated by 3-D analysis. Synergy may or may not have been detected depending of the endpoint chosen by the investigator.

Results of the CI plot depend on the molar ratio of APTS and ACV chosen for the experiment. This is a result of the experimental design, which samples points at an arbitrary fixed ratio, yielding data points that lie on a diagonal across a doseresponse surface. Consequently, the exact nature of the interaction detected depends on the region that the diagonal intersects. As an example, when the $\mathrm{CI}$ is plotted at a ACV:APTS molar ratio of 3, the data points used to calculate the CI lie along a diagonal which intersects a region of antagonism and thus reveals antagonistic interactions (Fig. 6F). Alternatively, if an ACV:APTS molar ratio of 0.3 is used, the data points used in the calculations fall on a diagonal which intersects the region of potent synergy and consequently, the CI plot reveals synergistic interactions at high endpoints. The $\mathrm{CI}$ therefore may or may not completely disclose the drug interactions and depends on the molar ratio chosen by the investigator.

\section{Advantages of 3-D analysis}

The three dimensional method has many advantages over the 2-D approximations currently used to examine drug-drug interactions. First, the dose-response surface can be directly visualized and plotted. Second, predicted additive effects can be calculated using either the similar site or dissimilar site assumptions of additivity. The resulting additive surface can be subtracted from the experimental surface to reveal areas of synergy and antagonism. Third, the synergy and antagonism can be quantitated which allows the synergy of different drug combinations to be compared. Fourth, the data can be analyzed to reveal which observed drug interactions are statistically significant. Fifth, the methods of data acquisition and analysis are efficient and facilitate the routine analysis of drug combinations. Finally, the shape of the synergy plot is a characteristic of the particular drug combination used. Three dimensional analysis may reveal interesting stoichiometric or other relationships which may hint at the underlying mechanisms of synergy.

Drug-drug interactions are inherently defined by a 3-D dose-response surface When 2-D methods are used to analyze these interactions, one variable must be held constant, thus making the analysis incomplete. Results analyzed with currently used 2-D methods may mislead investigators if they are unaware of the 3-D implications of their 2-D analytical method. It is therefore crucial that the interactions between drugs be viewed in their proper 3-D context as important synergistic or antagonistic interactions could be underestimated or missed entirely by traditional 2-D methods.

Ours is not an original model. It is at least 100 years old, but researchers have been largely unaware of the the extremely important 3-D context of their analytical methods. This oversight took place presumably because of the technical difficulties inherent in 3-D analyses in general. Now, through the advances in microcomputers 
and sophisticated analytical programs, the technology to analyze drug interactions in their proper 3-D context is available to most investigators. This creates a unique opportunity to thoroughly explore the characteristics of combinations of antiviral drugs. The rigorous analyses performed by these new methods may yield new insights about the complex interactions between drugs and the principles of chemotherapeutic synergy.

\section{Acknowledgement}

The expert assistance of Professor Charles J. Kowalski in statistical interpretations is gratefully acknowledged. This work was supported by Public Health Service Grant DE-08510 from the National Institute of Dental Research.

\section{References}

Ahmed, A.L.M. and Tyrell, D.A.J. (1986) Synergism between anti-rhinovirus antivirals: various human interferons and a number of synthetic compounds. Antiviral Res. 6, 241-252.

Allen, L.B., Vanderslice, L.K., Fingal, C.F., McCright, F.H., Harris, E.F. and Cook, P.D. (1982) Evaluation of the anti-herpesvirus drug combinations: virazole plus arabinofuranosylhypoxanthine and virazole plus arabinofuranosyladenine. Antiviral Res. 2, 203-216.

Barer, M.R., Lyon, H. and Draser, B.S. (1986) Quantitation of dye binding by cell monolayers in a microtiter system. Histochem. J. 18, 122-128.

Bauer, D.J. (1954) The antiviral and synergistic actions of isatin thiosemicarbazone and certain phenoxypyrimidines in vaccinia infection in mice. Br. J. Exp. Pathol. 36, 105-114.

Berenbaum, M.C. (1976) Synergy, additivism and antagonism in immunosuppression. Clin. Exp. Immunol. 28, 1-18.

Berenbaum, M.C. (1981) Criteria for analyzing interactions between biologically active agents. Adv. Cancer Res. 35, 269-332.

Berenbaum, M.C. (1985) The expected effect of a combination of agents: the general solution. J. Theor. Biol. 114, 413-431.

Berenbaum, M.C. (1988) Isobolographic, algebraic, and search methods in the analysis of multiagent synergy. J. Am. Coll. Toxicol. 7, 927-938.

Carter, W.H. Jr., Gennings, C., Staniswalis, J.G., Campbell, E.D. and White, K.L. Jr. (1988) A statistical approach to the construction and analysis of isobolograms. J. Am. Coll. Toxicol. 7, 937-973.

Carter, W.H. Jr., Jones, D.E. and Carchman, R.A. (1985) Application of response surface methods for evaluating interactions of soman, atropine, and pralidioxime chloride. Fundam. Appl. Toxicol. 5, S232-S241.

Chan, E.L. and Zabransky, R.J. (1987) Determination by two methods with eight antimicrobial combinations against tobramycin-succeptible and tobramycin-resistant strains of pseudomonas. Diagn. Microbiol. Infect. Dis. 6, 157-164.

Chou, T.-C. and Talalay, P. (1980) Generalized equations for the analysis of inhibitors of mechaelismenton and higher order kinetic systems with two or more mutually exclusive and nonexclusive inhibitors. Eur. J. Biochem. 249, 2928-2931.

Chou, T.-C. and Talalay, P. (1983) Analysis of combined drug effects: a new look at a very old problem. Trends Pharmacol. Sci. 4, 450-454.

Chou, T.-C. and Talalay, P. (1984) Quantitative analysis of dose-effect relationships: the combined effects of multiple drugs or enzyme inhibitors. Adv. Enzyme Regul. 22, 27-55.

Elion, G.B., Singer, S. and Hitchings, G.H. (1954) Antagonists of nucleic acid derivatives. J. Biol. Chem. 208, $477-488$.

Forti, R.L., Schuffman, S.S., Davies, H.A. and Mitchell W.M. (1986) Objective antiviral assay of the 
interferons by computer assisted data collection and analysis. Methods Enzymol. 119, 533-541.

Fraser, T.R. (1872) The antagonism between the actions of active substances. Br. Med. J. 457-459 and $485-487$

Fraser-Smith, E.B., Eppstein, D.A., Marsh, Y.V. and Matthews, T.R. (1985) Enhanced efficacy of the acyclic nucleoside 9-(1,3-dihydroxy-2-propoxymethyl)guanine in combination with gamma interferon against herpes simplex virus type 2 in mice. Antiviral Res. 5, 137-144.

Freestone, D.S. (1985) The need for new antiviral agents. Antiviral Res. 5, 307-324.

Gessner, P.K. (1988) A straightforward method for the study of drug interactions: an isobolographic analysis primer. J. Am. Coll. Toxicol. 7, 987-1012.

Gershwin, M.E. and Smith, N.T. (1973) Interaction between drugs using three-dimensional isobolographic interpretation. Arch. Int. Pharmacodyn. 201, 154-161.

Goldwin, A. and Mantel, N. (1957) The employment of combinations of drugs in the chemotherapy of neoplasia: a review. Cancer Res. 17, 635-654.

Hall, M.J. and Duncan, I.B. (1988) Antiviral drug and interferon combinations. In: H.J Fields (ed), Antiviral Agents: The Development and Assessment of Antiviral Chemotherapy, Vol. II, pp. 29-84. CRC Press, Boca Raton, FL.

Hartshorn, K.L., Vogt, M.W., Chou, T.-C., Blumberg, R.S., Byington, R., Schooley, R.T. and Hirsch, M.S. (1987) Synergistic inhibition of human immunodeficiency virus in vitro by azidothymidine and recombinant alpha a interferon. Antimicrob. Agents Chemother. 31, 168-172.

Huggins, J.W., Robins, R.K. and Canonico, P.G. (1984) Synergistic antiviral eftects of ribavirin and the c-nucleoside analogs tiazofurin and selenazofurin against togaviruses, bunyaviruses, and arenaviruses. Antimicrob. Agents Chemother. 26, 476-480.

Hunt, R.J. (1986) Percent agreement, Pearson's correlation, and kappa as measures of inter-examiner reliability. J. Dent. Res. 65, 128-130.

Jackson, R.C. (1984) A kinetic model of regulation of the deoxyribonucleoside triphosphate pool composition. Pharmac. Ther. 24, 279-301.

Jawetz, E. (1968) The use of combinations of antimicrobial drugs. Ann. Rev. Pharmacol. 8, 151-170.

Johnson, J.C. and Attanasio, R. (1987) Synergistic inhibition of anatid herpesvirus replication by acyclovir and phosphonocompounds. Intervirology 28, 89-99.

Karlsson, A. and Harmenberg, J. (1988) Effects of ribonucleotide reductase inhibition on pyrimidine deoxynucleotide metabolism in acyclovir-treated cells infected with herpes simplex virus type 1. Antimicrob. Agents Chemother. 32, 1100-1102.

Kirsi, J.J., McKerron, P.A., Burns, N.J., IIl, North, J.A., Murray, B.K. and Robins, R.K. (1984) Broadspectrum synergistic antiviral activity of selenazofurin and ribavirin. Antimicrob. Agents Chemother. $26,466-475$.

Klein, R.J. (1975) Isolation of herpes simplex virus clones and drug resistant mutants in microcultures. Arch. Virol. 49, 73-80.

Lam, G.K.Y. (1987) The interaction of radiations of different LET. Phys. Med. Biol. 32, 1291-1309.

Landini, M. P. and Baldassarri, B. (1983) A rapid antiviral drug test by enzyme-linked immunoassay. Diag. Immunol. 1, 284-287.

Langlois, M., Allard, J.P., Nugier, F. and Aymard, M. (1986) A rapid and automated colorimetric assay for evaluating the sensitivity of herpes simplex strains to antiviral drugs. J. Biol. Stand. 14, 201-211.

Loewe, S. (1928) Die Quantitations probleme der Pharmakologie. Ergebn. Physiol. 27, 47-187.

Loewe, S. (1938) Coalative actions of combined drugs. J. Pharmacol. 63, 24.

Loewe, S. (1953) The problem of synergism and antagonism of combined drugs. Arzneim. Forsch. 3 , $285-320$.

Loewe, S. (1954) Potentiation of drug-produced ejaculation by $\beta$-diethylaminoethyldiphenylpropylacetate (SKF No. 525A). J. Pharmacol. 110, 271-276.

Loewe, S. (1957) Antagonism and antagonists. Pharmacol. Rev. 9, 237-242.

London, J.W. Shaw, L.M., Theodorsen, L. and Stromme, J.H. (1982) Application of response surface methodology to the assay of gamma-glutamyitransferase. Clin. Chem. 5, 1140-1143.

MacKay, D. (1981) An analysis of functional antagonism and synergism. Br. J. Pharmacol. 73, 127-134.

Maddox, I.S. and Richert, S.H. (1977) Use of response surface methodology for the rapid optimalization of microbiological media. J. Appl. Bacteriol. 43, 197-204.

Mead, R. and Pike, D.J. (1975) A review of response surface methodology from a biometric viewpoint. 
Biometrics 31, 803-851.

Palatini, P. (1982) The interaction between full and partial inhibitors acting on a single enzyme. A theoretical analysis. Mol. Pharmacol. 24, 30-41.

Potmesil, M., Israel, M., Kirschenbaum, S. and Silber, R. (1986) Effects of N-trifluoroacetyladriamycin14-O-hemiadipate and radiation on L1210 cells. Rad. Res. 105, 147-157.

Rabalais, G.P., Levin, M.J. and Berkowitz, F.E. (1987) Rapid herpes simplex susceptibility testing using an enzyme-linked immunosorbant assay performed in situ on fixed virus infected monolayers. Antimicrob. Agents Chemother. 31, 946-948.

Sandstrom, E.G. and Kaplan, J.C. (1987) Antiviral therapy in AIDS clinical pharmacological properties and therapeutic experience to date. Drugs 34, 372-390.

Schinazi, R.F., Chou, T.-C., Scott, R.T., Yao, X. and Nahmias, A.J. (1986) Delayed treatment with combinations of antiviral drugs in mice infected with herpes simplex virus and application of the median effect method of analysis. Antimicrob. Agents Chemother. 30, 491-498.

Shipman, C., Jr. (1969) Evaluation of 4-(2-hydroxyethyl)-1-piperazineëthanesulfonic acid (HEPES) as a tissue culture buffer. Proc. Soc. Exp. Biol. 130, 305-310.

Skipper, H.E. (1974) Combination therapy: some concepts and results. Can. Chemother. Rep. 4, $137-145$.

Solana, R.P., Chinchilli, V.M., Wilson, J., Jr., W.H. and Carchman, R.A. (1986) Estimation and testing of the concentration-response surfaces associated with multiple agent combinations. Toxicol. App. Pharmacol. 85, 231-238.

Spector, T., Averett, D.R., Nelson, D.J., Lambe, C.U., Morrison, R.W., Jr., St.Claire, M.H. and Furman, P.A. (1985) Potentiation of antiherpetic activity of acyclovir by ribonucleotide reductase inhibition. Proc. Natl. Acad. Sci. USA 82, 42544257.

Spector, T., Harrington, J.A., Morrison, R., Jr., Lambe, C.U., Nelson, D.J., Averett, D.R. Biron, K. and Furman, P.A. (1989) 2-Acetylpyridine 5-[(dimethylamino)thiocarbonyl]-thiocarbonohydrazone (A1110U), a potent inactivator of ribonucleotide reductases of herpes simplex and varicella-zoster viruses and a potentiator of acyclovir. Proc. Natl. Acad. Sci. USA 86, 1051-1055.

Steel, G.G. and Peckham, M.J. (1979) Exploitable mechanisms in combined radiotherapy- chemotherapy: the concept of additivity. J. Radiation Oncol. Biol. Phys. 5, 85-91.

Streifel, J.A. and Howell, S.B. (1981) Synergistic interaction between 1- $\beta$-D-arabinofuranosylcytosine, thymidine, and hydroxyurea against human B cells and leukemic blasts in vitro. Proc. Natl. Acad. Sci. 78, 5132-5136.

Sühnel, J. (1990) Evaluation of synergism and antagonism for the combined action of antiviral agents. Antiviral Res. 13, 23-40.

Turk, S.R., Shipman, C., Jr. and Drach, J.C. (1986a) Selective inhibition of herpes simplex virus ribonucleoside diphosphate reductase by derivitives of 2-acetylpyridine thiosemicarbazone. Biochem. Pharmacol. 35, 1539-1545.

Turk, S.R., Shipman, C., Jr. and Drach, J.C. (1986b) Structure activity relationships among $\alpha$-(N)heterocyclic acyl thiosemicarbazones and related compounds as inhibitors of herpes simplex virus type 1 specified ribonucleoside diphosphate reductase. J. Gen. Virol. 67, 1625-1632.

Veldstra, H. (1956) Synergism and potentiation with special reference to the combination of structural analogues. Pharmacol. Rev. 8, 339-388.

Voght, M.W., Hartshorn, K.L., Furman, P.A., Chou, T.-C., Fyfe, J.A., Coleman, L.A., Crumpacker, C., Schooley, R.T. and Hirsch, M.S. (1987) Ribaviroin antagonizes the effect of azidothymidine on HIV replication. Science 235, 1376-1379.

Webb, J.L. (1963) Enzyme and metabolic inhibitors, Vol. 1, pp. 55-79 and 488-512. Academic Press, New York.

Yeh, T.-J., McBride, P.T., Overall, J.C. Jr., and Green, J.A. (1982) Automated, quantitative cytopathic effect reduction assay for interferon. J. Clin. Microbiol. 16, 413-415 


\section{Appendix}

\section{Derivation of additivity equations}

Similar site inhibitors The general equation for the isobologram at an endpoint of $P$ percent with $D_{1}$ and $D_{2}$ equal to the doses of drugs 1 and 2 respectively, and $\left(I C_{\mathrm{p}}\right)_{1}$ and $\left(I C_{\mathrm{p}}\right)_{2}$ equal to the inhibitory concentrations of drugs 1 and 2 required to produce $P$ percent inhibition:

$1=\frac{D_{1}}{\left(I C_{p}\right)_{1}}+\frac{D_{2}}{\left(I C_{p}\right)_{2}}$

assuming that the dose-response curves are linear with the same $I C_{\mathrm{p}}$, then the expressions

$\frac{D_{1}}{\left(I C_{p}\right)_{1}}$ and $\frac{D_{2}}{\left(I C_{p}\right)_{2}}$

represent the fractional inhibition of $P$ by drugs 1 and 2 respectively which can be expressed as percent inhibition by multiplying by $P$, yielding

$P=P \frac{D_{1}}{\left(I C_{p}\right)_{1}}+P \frac{D_{2}}{\left(I C_{p}\right)_{2}}$

substituting $Z$ for the total inhibition produced by combinations at all endpoints, and $X$ for the inhibition produced by drug 1 and $Y$ for the inhibition produced by drug 2, eq. 2 becomes

$Z=X+Y$

which confirms the similar site assumption that the total inhibition is the sum of the individual inhibitions.

Dissimilar site inhibitors Chou and Talalay's combination index equation for mutually nonexclusive drugs as derived from classical enzyme kinetics (Chou and Talalay, 1984), with $\left(f_{\mathrm{a}}\right)$ and $\left(f_{\mathrm{u}}\right)$ equal to the fractions affected, and fractions unaffected respectively, with subscripts designating the drug or combination used. This expression is equivalent to Webb's fractional inhibitory concentration equation which is simply another form of equation 10.

$\frac{\left(f_{a}\right)_{1,2}}{\left(f_{u 1}\right)_{1,2}}=\frac{\left(f_{a}\right)_{1}}{\left(f_{u}\right)_{1}}+\frac{\left(f_{a}\right)_{2}}{\left(f_{u}\right)_{2}}+\frac{\left(f_{a}\right)_{1}\left(f_{a}\right)_{2}}{\left(f_{u}\right)_{1}\left(f_{1}\right)_{2}}$

substituting,

$X=\left(\mathrm{f}_{\mathrm{a}}\right)_{1}=$ inhibition produced by drug $\mathrm{X}$

$Y=\left(\mathrm{f}_{\mathrm{i}}\right)_{2}=$ inhibition produced by drug $\mathrm{Y}$

$Z=\left(\mathrm{f}_{\mathrm{a}}\right)_{1,2}=$ inhibition produced by the combination

$\left(\mathrm{f}_{12}\right)=\left[1-\left(\mathrm{f}_{\mathrm{a}}\right)\right]$

$\frac{Z}{1-Z}=\frac{X}{1-X}+\frac{Y}{1-Y}+\frac{X Y}{(1-X)(1-Y)}$

finding a common denominator and simplifying,

$$
\begin{aligned}
& \frac{Z}{1-Z}=\frac{X(1-Y)+Y(1-X)+X Y}{(1-X)(1-Y)} \\
& \frac{Z}{1-Z}=\frac{X+Y-X Y}{1-X-Y+X Y} \\
& Z(1-X-Y+X Y)=(X+Y-X Y)(1-Z) \\
& Z(1-X-Y+X Y+X+Y-X Y)=X+Y-X Y \\
& Z=X+Y+(1-X)
\end{aligned}
$$

This confirms the dissimilar site assumption of additivity. 
Additivity for mutually exclusive inhibitors Chou's combination index equation for mutually exclusive drugs (Chou and Talalay, 1984), with $\left(f_{\mathrm{a}}\right)$ and $\left(f_{u}\right)$ equal to the fractions affected, and fractions unaffected respectively, with subscripts designating the drug or combination used.

$\frac{\left(f_{a}\right)_{1,2}}{\left(f_{u}\right)_{1,2}}=\frac{\left(f_{a}\right)_{1}}{\left(f_{u}\right)_{1}}+\frac{\left(f_{a}\right)_{2}}{\left(f_{u}\right)_{2}}$

substituting

$X=\left(\mathrm{f}_{\mathrm{a}}\right)_{1}=$ inhibition produced by drug $\mathrm{X}$

$Y=\left(\mathrm{f}_{\mathrm{a}}\right)_{2}=$ inhibition produced by drug $\mathrm{Y}$

$Z=\left(\mathrm{f}_{\mathrm{a}}\right)_{1,2}=$ inhibition produced by the combination

$\left(f_{u}\right)=\left[1-\left(f_{a}\right)\right]$

$\frac{Z}{1-Z}=\frac{X}{1-X}+\frac{Y}{1-Y}$

finding a common denominator and simpliíying,

$\frac{Z}{1-Z}=\frac{X(1-Y)+Y(1-X)}{(1-X)(1-Y)}$

$Z(1-X-Y+X Y)=(X+Y-2 X Y)(1-Z)$

$Z(1-X-Y+X Y+X+Y-2 X Y)=X+Y-2 X Y$

$Z(1-X Y)=X+Y-2 X Y$

$Z=\frac{X+Y-2 X Y}{1-X Y}$

Note that eq. 17 is not equivalent to eq. 3 as proposed by Chou and Talalalay (also see Fig. 4A-C). 
